\title{
Reconstruction of Multimodal Distributions for Hybrid Moment-based Chemical Kinetics Supporting Information
}

\author{
Alexander Andreychenko ${ }^{1}{ }^{*}$ Linar Mikeev $^{1}$, and Verena Wolf ${ }^{1}$ \\ ${ }^{1}$ Computer Science Department, Saarland University, Saarbrucken, Germany 66123
}

\begin{abstract}
The stochastic dynamics of biochemical reaction networks can be accurately described by discrete-state Markov processes where each chemical reaction corresponds to a state transition of the process. Due to the largeness problem of the state space, analysis techniques based on an exploration of the state space are often not feasible and the integration of the moments of the underlying probability distribution has become a very popular alternative. In this paper the focus is on a comparison of reconstructed distributions from their moments obtained by two different moment-based analysis methods, the method of moments (MM) and the method of conditional moments (MCM). We use the maximum entropy principle to derive a distribution that fits best to a given sequence of (conditional) moments. For the two gene regulatory networks that we consider we find that the MCM approach is more suitable to describe multimodal distributions and that the reconstruction of marginal distributions is more accurate if conditional distributions are considered.
\end{abstract}

Keywords: Chemical Master Equation, Moment Closure, Method of Conditional Moments, Maximum Entropy.

PACS: 02.50.Ga, 87.18.Vf, 02.50.Cw

MSC: 60J22, 44A60, 37N25

Appendix A: Supporting Information

In the following sections we first describe in detail how the moment equations are obtained (Section 1 and 2) and how we approximate the support of the distribution (Section 3). In Section 4 we then discuss the differences that arise during the reconstruction of distributions with two instead of only one dimension, and in Section 5 we discuss the details of the reconstruction for the distributions of the case studies introduced before. More numerical results for the two case studies are then provided in Section 6

\section{Method of Moments}

For the time derivative of the expectation of a function $f: \mathbb{N}_{0}^{n} \rightarrow \mathbb{R}^{n}$ applied to the vector of species, we directly get from Eq. (1)

$$
\begin{aligned}
\frac{d}{d t} & E(f(\vec{X}(t)))=\sum_{x} f(x) \frac{d}{d t} p(\vec{x}, t) \\
& =\sum_{j=1}^{m} E\left(\alpha_{j}(\vec{X}(t))\left(f\left(\vec{X}(t)+v_{j}\right)-f(\vec{X}(t))\right)\right) .
\end{aligned}
$$

For $f(\vec{x})=\vec{x}$ this yields a system of equations for the population means

$$
\frac{d}{d t} E(\vec{X}(t))=\sum_{j=1}^{m} v_{j} E\left(\alpha_{j}(\vec{X}(t))\right) .
$$

Note that the system of ODEs in Eq. (A2) is only closed if at most monomolecular reactions $\left(\sum_{i=1}^{n} \ell_{j, i} \leq 1\right)$ are in-

*Corresponding author. Email: alexander.andreychenko@uni-saarland.de volved. Otherwise $E\left(\alpha_{j}(\vec{X}(t))\right)$ involves moments of the second order. However, in this case we can approximate the unknown second order moments, say $E\left(X_{i}(t) \cdot X_{i^{\prime}}(t)\right)$ if the reaction is of the form $S_{i}+S_{i^{\prime}} \rightarrow \ldots, i \neq i^{\prime}$, either by assuming that the covariance is zero, which gives $E\left(X_{i}(t) \cdot X_{i^{\prime}}(t)\right)=E\left(X_{i}(t)\right) \cdot E\left(X_{i^{\prime}}(t)\right)$ or by extending the system in (A2) with additional equations for the second moments. The general strategy is to replace $\alpha_{j}(\vec{X}(t))$ by a Taylor series about the mean $E(\vec{X}(t))$. Let us write $\mu_{i}(t)$ for $E\left(X_{i}(t)\right)$ and $\vec{\mu}(t)$ for the vector with entries $\mu_{i}(t)$, $1 \leq i \leq n$. Then

$$
\begin{aligned}
& E\left(\alpha_{j}(\vec{X})\right)=\alpha_{j}(\vec{\mu})+\frac{1}{1 !} \sum_{i=1}^{n} E\left(X_{i}-\mu_{i}\right) \frac{\partial}{\partial x_{i}} \alpha_{j}(\vec{\mu}) \\
& +\frac{1}{2 !} \sum_{i=1}^{n} \sum_{k=1}^{n} E\left(\left(X_{i}-\mu_{i}\right)\left(X_{k}-\mu_{k}\right)\right) \frac{\partial^{2}}{\partial x_{i} \partial x_{k}} \alpha_{j}(\vec{\mu}) \\
& +\ldots
\end{aligned}
$$

where we omitted $t$ in the equation to improve readability. Note that $E\left(X_{i}(t)-\mu_{i}\right)=0$ and since we restrict to reactions that are at most bimolecular with mass action kinetics, all terms of order three or more disappear. The derivation of moments for general kinetics is presented in [1].

$$
\begin{gathered}
\text { By letting } C_{i k} \text { be the covariance } \\
E\left(\left(X_{i}(t)-\mu_{i}\right)\left(X_{k}(t)-\mu_{k}\right)\right) \text {, we get } \\
E\left(\alpha_{j}(\vec{X})\right)=\alpha_{j}(\vec{\mu})+\frac{1}{2} \sum_{i=1}^{n} \sum_{k=1}^{n} C_{i k} \frac{\partial^{2}}{\partial x_{i} \partial x_{k}} \alpha_{j}(\vec{\mu}) .
\end{gathered}
$$

Next, we derive an equation for the covariances by first exploiting the relationship

$$
\begin{aligned}
& \frac{d}{d t} C_{i k}=\frac{d}{d t} E\left(X_{i} X_{k}\right)-\frac{d}{d t}\left(\mu_{i} \mu_{k}\right) \\
& =\frac{d}{d t} E\left(X_{i} X_{k}\right)-\left(\frac{d}{d t} \mu_{i}\right) \mu_{k}-\mu_{i}\left(\frac{d}{d t} \mu_{k}\right)
\end{aligned}
$$


and if we couple this equation with the equations for the means, the only unknown term that remains is the derivative $\frac{d}{d t} E\left(X_{i} X_{k}\right)$ of the second moment. For this we can use the same strategy as before, i.e., from Eq. A1 we get

$$
\begin{aligned}
& \frac{d}{d t} E\left(X_{i} X_{k}\right)=\sum_{j=1}^{m}\left(v_{j, i} v_{j, k} E\left(\alpha_{j}(\vec{X})\right)\right. \\
& \left.\quad+v_{j, k} E\left(\alpha_{j}(\vec{X}) X_{i}\right)+v_{j, i} E\left(\alpha_{j}(\vec{X}) X_{k}\right)\right),
\end{aligned}
$$

where $v_{j, i}$ and $v_{j, k}$ are the corresponding entries of the vector $v_{j}$. Clearly, we can use Eq. (A4) for the term $E\left(\alpha_{j}(\vec{X})\right)$, while the terms $E\left(\alpha_{j}(\vec{X}) X_{i}\right)$ and $E\left(\alpha_{j}(\vec{X}) X_{k}\right)$ have to be replaced by the corresponding Taylor series about the mean. Let $f_{j}(\vec{x}):=\alpha_{j}(\vec{x}) x_{i}$. Similar to Eq. (A4), we get that $E\left(\alpha_{j}(\vec{X}) X_{i}\right)$ equals

$$
\begin{aligned}
& \alpha_{j}(\vec{\mu}) \mu_{i}+\frac{1}{1 !} \sum_{i=1}^{n} E\left(X_{i}-\mu_{i}\right) \frac{\partial}{\partial x_{i}} f_{j}(\vec{\mu}) \\
& +\frac{1}{2 !} \sum_{i=1}^{n} \sum_{k=1}^{n} E\left(\left(X_{i}-\mu_{i}\right)\left(X_{k}-\mu_{k}\right)\right) \frac{\partial^{2}}{\partial x_{i} \partial x_{k}} f_{j}(\vec{\mu}) \\
& +\ldots
\end{aligned}
$$

Here, it is important to note that moments of order three come into play, since derivatives of order three of $f_{j}(\vec{x})=$ $\alpha_{j}(\vec{x}) x_{i}$ may be nonzero. It is possible to take these terms into account by deriving additional equations for moments of order three and higher. Obviously, these equations will then include moments of even higher order such that theoretically we end up with an infinite system of equations. However, a popular strategy is to close the equations by assuming that all moments of order $>M$ that are centered around the mean are equal to zero. E.g. if we choose $M=2$, then we can simply use the approximation

$$
\begin{aligned}
& E\left(\alpha_{j}(\vec{X}) X_{i}\right) \approx \alpha_{j}(\vec{\mu}) \mu_{i} \\
& +\frac{1}{2 !} \sum_{i=1}^{n} \sum_{k=1}^{n} E\left(\left(X_{i}-\mu_{i}\right)\left(X_{k}-\mu_{k}\right)\right) \frac{\partial^{2}}{\partial x_{i} \partial x_{k}} f_{j}(\vec{\mu}) .
\end{aligned}
$$

Other methods can be used to close the system of equations, including derivative matching and zero cumulants closure [6], as well as those that make assumptions about the distribution of the moments [3, 4, 7]

The given approximation is then inserted into Eq. A6 and the result is used to replace the term $\frac{d}{d t} E\left(X_{i} X_{k}\right)$ in Eq. A5. Finally, we can integrate the time evolution of the means and that of the covariances and variances.

Example 2. We apply the moment closure technique described above to the gene expression system from Example 1. When we consider only the moments up to second order, the corresponding equations for the average number of molecules are, for instance, given by

$$
\begin{aligned}
& \frac{d}{d t} \mu_{D_{\mathrm{off}}}=\tau_{\mathrm{off}} \mu_{D_{\mathrm{on}}}-E\left(\tau_{\mathrm{on}}^{p} X_{D_{\mathrm{off}}} X_{P}\right) \\
& \frac{d}{d t} \mu_{D_{\mathrm{on}}}=\tau_{\mathrm{on}} \mu_{D_{\mathrm{off}}}+E\left(\tau_{\mathrm{on}}^{p} X_{D_{\mathrm{off}}} X_{P}\right) \\
& \frac{d}{d t} \mu_{R}=k_{r} \mu_{D_{\mathrm{on}}}-\gamma_{r} \mu_{R} \\
& \frac{d}{d t} \mu_{P}=k_{p} \mu_{R}-\gamma_{p} \mu_{P}
\end{aligned}
$$

where $\mu_{D_{\text {off }}}, \mu_{D_{\text {on }}}$ are the expected numbers of $D_{\text {off }}$ and $D_{\text {on }}$, respectively, and $\mu_{R}, \mu_{P}$ are the expected numbers of mRNA and proteins.

Next we compare the obtained moments with those computed via a direct numerical integration of the CME (Table I). We consider the following three cases. The moment closure approximation is carried out using all moments up to order 4,6 , and 8 . For each case we list the number of moment equations, the running time, and the relative errors in the first four moments (columns 4-7). The relative error for the moments of order $l$ for species $i$ is given by $\epsilon_{l}^{r}=\max _{1 \leq i \leq n}\left|\mu_{l}^{(i)}-\check{\mu}_{l}^{(i)}\right| / \check{\mu}_{l}^{(i)}$, where $\mu_{l}^{(i)}$ and $\check{\mu}_{l}^{(i)}$ are the values of the moments computed using the moment closure and obtained with a direct integration of the CME.

Please note that in the reconstruction procedure we do not use the moment of the highest order. For example, if we approximate moments up to order 6 , then the highest order that is taken into account during the reconstruction is 5 (which corresponds to the case $M=5$, cf. Sect. 6) because of the high sensitivity of the numerical procedure even to the small absolute error in the moment approximation.

\section{Method of Conditional Moments}

We first decompose the chemical populations described by $\vec{X}(t)$ into small and large populations. Here we assume that this decomposition is static. However, it is obvious that during the integration over time, we can (after reconstructing the joint distribution) choose a different decomposition for the remaining time. From what size on a population should be considered as small is typically dependent on the amount of main memory that is available and on the maximum order of the moments that we consider for the large populations. Note that considering conditional moments

TABLE I: Moment closure approximation results for the gene expression system

\begin{tabular}{ccccccc}
\hline $\begin{array}{c}\text { moment } \\
\text { closure } \\
\text { order }\end{array}$ & $\begin{array}{c}\text { time equ. } \\
\text { \# }(\mathrm{sec})\end{array}$ & $\begin{array}{c}\text { error } \\
\text { ord. 1 } \\
\text { moments }\end{array}$ & $\begin{array}{c}\text { error } \\
\text { ord. 2 } \\
\text { moments }\end{array}$ & $\begin{array}{c}\text { error } \\
\text { ord. 3 } \\
\text { moments }\end{array}$ & $\begin{array}{c}\text { error } \\
\text { ord. 4 } \\
\text { moments }\end{array}$ \\
\hline 4 & 70 & 1 & $8 \cdot 10^{-6}$ & $8.3 \cdot 10^{-5}$ & $9.6 \cdot 10^{-5}$ & $8.24 \cdot 10^{-4}$ \\
\hline 6 & 209 & 25 & $2 \cdot 10^{-6}$ & $2 \cdot 10^{-6}$ & $1 \cdot 10^{-5}$ & $3.6 \cdot 10^{-5}$ \\
\hline 8 & 494 & 3726 & $1 \cdot 10^{-6}$ & $2 \cdot 10^{-6}$ & $2 \cdot 10^{-6}$ & $4 \cdot 10^{-6}$ \\
\hline
\end{tabular}


yields a smaller number of equations if the order of the considered moments is high. The reason is that the number of equations for representing the dynamics of the small populations does not increase as the order of considered conditional moments increases. Also, for many systems the decomposition is obvious, as the small populations are exactly those that have a maximal size of, say, less than 10 (because they represent binding sites) and the large populations count protein numbers which may become rather large.

Formally, we write the random vector $\vec{X}(t)$ at time $t$ as $\vec{X}(t)=(\vec{Y}(t), \vec{Z}(t))$, where $\vec{Y}(t)$ corresponds to the small, and $\vec{Z}(t)$ to the large populations. Similarly, we write $\vec{x}=$ $(\vec{y}, \vec{z})$ for the states of the process and $v_{j}=\left(\hat{v}_{j}, \tilde{v}_{j}\right)$ for the change vectors, $j \in\{1, \ldots, m\}$. Again, the first component refers to the small and the second component to the large populations. Now, Eq. 1 becomes

$$
\begin{array}{r}
\frac{d p(\vec{y}, \vec{z})}{d t}=\sum_{j=1}^{m}\left(\alpha_{j}\left(\vec{y}-\hat{v}_{j}, \vec{z}-\tilde{v}_{j}\right) p\left(\vec{y}-\hat{v}_{j}, \vec{z}-\tilde{v}_{j}\right)\right. \\
\left.-\alpha_{j}(\vec{y}, \vec{z}) p(\vec{y}, \vec{z})\right)
\end{array}
$$

where we omitted the time parameter $t$ to improve readability. Next, we sum over all possible $\vec{z}$ to get the time evolution of the marginal distribution $\hat{p}(\vec{y})=\sum_{\vec{z}} p(\vec{y}, \vec{z})$ of the small populations.

$$
\begin{aligned}
& \frac{d}{d t} \hat{p}(\vec{y})= \\
& \sum_{\vec{z}} \sum_{j=1}^{m} \alpha_{j}\left(\vec{y}-\hat{v}_{j}, \vec{z}-\tilde{v}_{j}\right) p\left(\vec{y}-\hat{v}_{j}, \vec{z}-\tilde{v}_{j}\right) \\
& -\sum_{\vec{z}} \sum_{j=1}^{m} \alpha_{j}(\vec{y}, \vec{z}) p(\vec{y}, \vec{z})= \\
& \sum_{j=1}^{m} \hat{p}\left(\vec{y}-\hat{v}_{j}\right) E\left[\alpha_{j}\left(\vec{y}-\hat{v}_{j}, \vec{Z}\right) \mid Y=\vec{y}-\hat{v}_{j}\right] \\
& -\sum_{j=1}^{m} \hat{p}(\vec{y}) E\left[\alpha_{j}(\vec{y}, \vec{Z}) \mid \vec{Y}=\vec{y}\right]
\end{aligned}
$$

Note that in this small master equation that describes the change of the mode probabilities over time, the sum runs only over those reactions that modify $\vec{y}$, since for all other reactions the terms cancel out. Moreover, on the right side we have only mode probabilities of neighboring modes and conditional expectations of the continuous part of the reaction rate. For the latter, we can use a Taylor expansion about the conditional population means. Similar to Eq. A3, this yields an equation that involves the conditional means and centered conditional moments of second order (variances and covariances). Thus, in order to close the system of equations, we need to derive equations for the time evolution of the conditional means and centered conditional moments of higher order. Since the mode probability $p(\vec{y})$ may become zero, we first derive an equation for the evolution of the partial means (conditional means multiplied by the probability of the condition)

$$
\begin{aligned}
& \frac{d}{d t}(E[\vec{Z} \mid \vec{y}] p(\vec{y}))=\sum_{\vec{z}} \vec{z} \frac{d}{d t} p(\vec{y}, \vec{z}) \\
& \quad=\sum_{j=1}^{m} E\left[\left(\vec{Z}+\tilde{v}_{j}\right) \alpha_{j}\left(\vec{y}-\hat{v}_{j}, \vec{Z}\right) \mid \vec{y}-\hat{v}_{j}\right] p\left(\vec{y}-\tilde{v}_{j}\right) \\
& \quad-\sum_{j=1}^{m} E\left[\vec{Z} \alpha_{j}(\vec{y}, \vec{Z}) \mid \vec{y}\right] p(\vec{y}),
\end{aligned}
$$

where in the second line we applied Eq. A8 and simplified the result. The conditional expectations $E[(\vec{Z}+$ $\left.\left.\tilde{v}_{j}\right) \alpha_{j}\left(\vec{y}-\hat{v}_{j}, \vec{Z}\right) \mid \vec{y}-\hat{v}_{j}\right]$ and $E\left[\vec{Z} \alpha_{j}(\vec{y}, \vec{Z}) \mid \vec{y}\right]$ are then replaced by their Taylor expansion about the conditional means such that the equation involves only conditional means and higher centered conditional moments [5]. For higher centered conditional moments, similar equations can be derived. If all centered conditional moments of order higher than $k$ are assumed to be zero, the result is a (closed) system of differential algebraic equations (algebraic equations are obtained whenever a mode probability $p(\vec{y})$ is equal to zero). However, it is possible to transform the system of differential algebraic equations into a system of (ordinary) differential equations after truncating modes with insignificant probabilities. Then we can get an accurate approximation of the solution after applying standard numerical integration methods. We construct the ODE system using the tool SHAVE* $^{*}$ which implements the trunca-

\begin{tabular}{|c|c|c|c|c|c|c|c|}
\hline $\begin{array}{l}\text { cond. } \\
\text { moment } \\
\text { closure } \\
\text { order }\end{array}$ & \# eq. & $\begin{array}{l}\text { time } \\
(\mathrm{sec})\end{array}$ & $\begin{array}{l}\text { error } \\
\text { cond. } \\
\text { probs. }\end{array}$ & $\begin{array}{c}\text { error } \\
\text { ord. } 1 \\
\text { cond. } \\
\text { moments }\end{array}$ & $\begin{array}{c}\text { error } \\
\text { ord. } 2 \\
\text { cond. } \\
\text { moments }\end{array}$ & $\begin{array}{c}\text { error } \\
\text { ord. } 3 \\
\text { cond. } \\
\text { moments }\end{array}$ & $\begin{array}{c}\text { error } \\
\text { ord. } 4 \\
\text { cond. } \\
\text { moments }\end{array}$ \\
\hline 4 & 30 & 1 & $7 \cdot 10^{-6}$ & $1 \cdot 10^{-5}$ & $2.86 \cdot 10^{-4}$ & $1.12 \cdot 10^{-3}$ & $6.98 \cdot 10^{-3}$ \\
\hline 6 & 56 & 2 & $6 \cdot 10^{-6}$ & $3.6 \cdot 10^{-5}$ & $5.9 \cdot 10^{-5}$ & $6.8 \cdot 10^{-5}$ & $2.18 \cdot 10^{-4}$ \\
\hline 8 & 90 & 9 & $2 \cdot 10^{-6}$ & $4.2 \cdot 10^{-5}$ & $6.2 \cdot 10^{-5}$ & $7.7 \cdot 10^{-5}$ & $9.1 \cdot 10^{-5}$ \\
\hline
\end{tabular}
tion based approach and solve it using MATLAB's ode 45 solver with the default error tolerance settings.

Example 3. We apply the method of conditional moments to the gene expression system from Example 1. The modes of the system are then given by the state of the DNA. The equations for the mode probabilities $\left(p_{\text {off }}, p_{\text {on }}\right)$ and the expected number of mRNA $\left(\mu_{R, \text { off }}, \mu_{R, \text { on }}\right)$ and proteins

TABLE II: Conditional moment closure approximation results for the gene expression system

* L. Mikeev, http://almacompute.mmci.uni-saarland.de/shave/ 
$\left(\mu_{P, \text { off }}, \mu_{P, \text { on }}\right)$ are as follows:

$$
\begin{aligned}
& \frac{d}{d t} p_{\text {off }}=\tau_{\text {on }} p_{\text {on }}-\left(\tau_{\text {off }}+\tau_{\text {on }}^{p} \mu_{P, \text { off }}\right) p_{\text {off }} \\
& \frac{d}{d t}\left(\mu_{R, \text { off }} p_{\text {off }}\right)=-\gamma_{r} \mu_{R, \text { off }} p_{\text {off }} \\
& \frac{d}{d t}\left(\mu_{P, \text { off }} p_{\text {off }}\right)=\left(k_{p} \mu_{R, \text { off }}-\gamma_{p} \mu_{P, \text { off }}\right) p_{\text {off }} \\
& \frac{d}{d t} p_{\text {on }}=\left(\tau_{\text {off }}+\tau_{\text {on }}^{p} \mu_{P, \text { off }}\right) p_{\text {off }}-\tau_{\text {on }} p_{\text {on }} \\
& \frac{d}{d t}\left(\mu_{R, \text { on }} p_{\text {on }}\right)=\left(k_{r}-\gamma_{r} \mu_{R, \text { on }}\right) p_{\text {on }} \\
& \frac{d}{d t}\left(\mu_{P, \text { on }} p_{\text {on }}\right)=\left(k_{p} \mu_{R, \text { on }}-\gamma_{p} \mu_{P, \text { on }}\right) p_{\text {on }}
\end{aligned}
$$

We computed the conditional moments and conditional probabilities of the running example (cf. Ex. 1 and Ex. 2) over time by considering moments up to the order of 4,6 , and 8 . For these three cases the number of equations, when compared to the method of moments (MM), are as follows:

\begin{tabular}{lccc}
\hline moment order $M$ & 4 & 6 & 8 \\
\hline \# equations for MM & 69 & 209 & 494 \\
\hline \# equations for MCM & 30 & 56 & 90 \\
\hline
\end{tabular}

The relative errors $\epsilon_{l}^{r}$ of the results of the method of conditional moments (MCM) are given in Table II. where we again compared to the results obtained via a direct numerical solution.

Our experiments show that the MCM performs much faster (due to the smaller number of equations) and still yields accurate approximation of the moments. For the chosen set of parameters the MCM tends to provide a better approximation for higher moments, whereas the MM approach is more accurate for lower moments when the same number of moments is considered. For example, in the case of 6 moments the maximum relative error for the first moments computed by the MM approach is $2 \cdot 10^{-6}$, compared to $3.2 \cdot 10^{-5}$ when computed using the MCM. At the same time, the maximum relative errors of the sixth moments are $6.5 \cdot 10^{-4}$ and $2 \cdot 10^{-4}$ for the MM and the MCM respectively. Note that the (unconditional) moments for the MCM are computed via multiplication of the conditional moments with the mode probabilities and sum over all possible conditions. We only consider non-central moments because the central moments introduce additional difficulties in the reconstruction framework.

We also consider another set of parameters for the gene expression kinetics. The rate constants are chosen $\left(\tau_{\text {on }}, \tau_{\text {off }}, k_{r}, k_{p}, \gamma_{r}, \gamma_{p}, \tau_{o n}^{p}\right)=$ $(0.05,0.05,10,1,4,1,0.015)$ as in [5]. For the initial states we simply use $x_{0,1}=(1,0,4,10)$ and $x_{0,2}=(1,0,4,10)$ with probabilities $P\left(x_{0,1}\right)=0.7$ and $P\left(x_{0,1}\right)=0.3$. The comparison of the moment values at time instant $t=10$ reveals that the MCM provides a much better approximation both for high and low order moments as opposed to the first parameter set. For instance, in the case of 6 moments the maximum relative error for the first moments computed by the MM approach is 0.14 whereas in the MCM approach the error is $7.5 \cdot 10^{-5}$. The maximum relative error of the sixth moments for the MM approach is 0.28 compared to 0.02 using the MCM.

\section{Approximation of the Support}

During the iteration we approximate the moments using Eq. (6), where we do not sum over all states $x \in \mathbb{N}_{0}$ but consider a subset $D=\left\{x_{L}, \ldots, x_{R}\right\} \subset \mathbb{N}_{0}$. Note that we have to find appropriate values for $x_{L}$ and $x_{R}$, since the iteration might fail to converge if the chosen value of $x_{R}$ is very large (and if $x_{L}=0$ ) as the conditional number of the matrix $\left(H+\gamma^{(\ell)} \cdot \operatorname{diag}(H)\right)$ is very large in this case. Thus, we make use of the results in [8] to find a region that contains the main part of the probability mass. We consider the roots of the function

$$
\Delta^{0}(w)=\left|\begin{array}{cccc}
\mu_{0} & \mu_{1} & \cdots & \mu_{k} \\
\vdots & & & \vdots \\
\mu_{k-1} & \mu_{k} & \cdots & \mu_{2 k-1} \\
1 & w & \cdots & w^{k}
\end{array}\right|
$$

where $k=\left\lfloor\frac{M}{2}\right\rfloor$, and $M$ is even. Let $W=\left\{w_{1}, \ldots, w_{k}\right\}$ be the set of the solutions of $\Delta^{0}(w)=0$, where $w_{1}<$ $\ldots<w_{k}$ are real and simple roots. The set $D^{(0)}=$ $\left\{x_{L}^{(0)}, \ldots, x_{R}^{(0)}\right\}$ with $x_{L}^{(0)}=\left\lfloor w_{1}\right\rfloor$ and $x_{R}^{(0)}=\left\lceil w_{k}\right\rceil$ is used as an initial guess for the approximated support when we start the optimization procedure. The final results $\lambda^{*}$ and $D^{*}$ of the iteration yields the distribution

$$
\tilde{q}(x)=\exp \left(-1-\sum_{k=0}^{M} \lambda_{k}^{*} x^{k}\right),
$$

which is an approximation of the marginal distribution $p .(x, t)=P(X .(t)=x)$, i.e. $p .(x, t) \approx \tilde{q}(x)$ if $x \in$ $D^{*}$ and $p .(x, t) \approx 0$ if $x \notin D^{*}$.

We can also account for the case of an odd number of moments. In addition to the function $\Delta^{0}(w)$ defined in Eq. A10, we also consider the function $\Delta^{1}(\eta)$

$$
\Delta^{1}(\eta)=\left|\begin{array}{ccc}
\mu_{1}-\eta_{1} \mu_{0} & \cdots & \mu_{z}-\eta_{1} \mu_{z-1} \\
\vdots & \vdots & \vdots \\
\mu_{z-1}-\eta_{1} \mu_{z-2} & \cdots & \mu_{2 z-2}-\eta_{1} \mu_{2 z-3} \\
1 & \cdots & \eta^{z-1}
\end{array}\right|,
$$

where $z=\left\lfloor\frac{M}{2}\right\rfloor+1$ and $w_{1}$ is the smallest root of the equation $\Delta^{0}(w)=0$. Again, let $W=\left\{w_{1}, \ldots, w_{k}\right\}$ be the set of the solutions of $\Delta^{0}(w)=0$ and $H=\left\{\eta_{1}, \ldots, \eta_{z}\right\}$ be the set of solutions of $\Delta^{1}(\eta)=0$, where all the elements of $W$ and $H$ are real and simple. The first approximation for the truncated support of the distribution is then given by the set $D^{(0)}=\left\{x_{L}^{(0)}, \ldots, x_{R}^{(0)}\right\}$ with $x_{L}^{(0)}=\left\lfloor\min \left(w_{1}, \eta_{1}\right)\right\rfloor$ and $x_{R}^{(0)}=\left\lceil\max \left(w_{k}, \eta_{z}\right)\right\rceil$. 
We extend the support until the relative change of the dual function becomes smaller than the threshold $\delta_{\Psi}$

$$
\left|\frac{\Psi\left(\lambda^{(\ell-1)}\right)-\Psi\left(\lambda^{(\ell)}\right)}{\Psi\left(\lambda^{(\ell)}\right)}\right|<\delta_{\Psi},
$$

where we choose $\delta_{\Psi}=10^{-4}$ for all case studies. If the inequality is not satisfied, we extend the support by adding new states in each iteration

$$
\left(x_{L}^{(\ell+1)}, x_{R}^{(\ell+1)}\right)=\left(\max \left(0, x_{L}^{(\ell)}-1\right), x_{R}^{(\ell)}+1\right)
$$

The final results $\tilde{\lambda}$ and $\hat{D}$ of the iteration yields the distribution $\tilde{q}(x)$ that approximates the marginal distribution of interest.

\section{Numerical Approach for the Two-dimensional Maximum Entropy Problem}

In the case of two-dimensional distributions, the maximum entropy problem is modified as follows. We consider a sequence of non-central moments $E\left(X^{r} X_{\circ}^{l}\right)=\mu_{r, l}$, $0 \leq r+l \leq M$, and the set $\mathcal{G}^{2}$ of all two-dimensional discrete distributions that satisfy the following constraints

$$
\sum_{x, y} x^{r} y^{l} g(x, y)=\mu_{r, l}, \quad 0 \leq r+l \leq M .
$$

Here $X$. and $X_{\circ}$ correspond to the populations of two different species, i.e. to two distinct elements of the random vector $\vec{X}(t)=\left(X_{1}(t), \ldots, X_{n}(t)\right)$ at some fixed time instant $t$. Similarly to the optimization problem (3), we seek the distribution $q \in \mathcal{G}^{2}$ that maximizes the entropy $H(g)$

$$
\begin{aligned}
q & =\arg \max _{g \in \mathcal{G}^{2}} H(g) \\
& =\arg \max _{g \in \mathcal{G}^{2}}\left(-\sum_{x, y} g(x, y) \ln g(x, y)\right)
\end{aligned}
$$

We then proceed similarly to the one-dimensional case. The general form of the solution for the maximum entropy problem is given by

$$
\begin{aligned}
q(x, y) & =\exp \left(-1-\sum_{0 \leq r+l \leq M} \lambda_{r, l} x^{r} y^{l}\right) \\
& =\frac{1}{Z} \exp \left(-\sum_{1 \leq r+l \leq M} \lambda_{r, l} x^{r} y^{l}\right),
\end{aligned}
$$

where the normalization constant $Z$ is calculated as

$$
Z=e^{1+\lambda_{0,0}}=\sum_{x, y} \exp \left(-\sum_{1 \leq r+l \leq M} \lambda_{r, l} x^{r} y^{l}\right) .
$$

We solve the optimization problem numerically similarly to the one-dimensional case. The vector $\lambda^{(\ell)}=$ $\left(\lambda_{0,1}, \lambda_{1,0}, \ldots, \lambda_{0, M}, \lambda_{M, 0}\right)$ is an approximation of the vector $\lambda$ in Eq. A15). The elements of the gradient vector are computed as $\partial \Psi / \partial \lambda_{r, l} \approx \mu_{r, l}-(1 / z) \widetilde{\mu}_{r, l}$, where $\widetilde{\mu}_{r, l}$ is approximated by

$$
\widetilde{\mu}_{r, l}=\sum_{x, y} x^{r} y^{l} \exp \left(-\sum_{1 \leq r+l \leq M} \lambda_{r, l} x^{r} y^{l} .\right),
$$

Here $r, l \in\{0, \ldots, 2 M\}$ and the sum is taken over all $(x, y) \in \mathbb{N}_{0}^{2}$. Finally, the elements of the Hessian matrix are computed as

$$
H_{r+u, l+v}=\frac{\partial^{2} \Psi}{\partial \lambda_{r, l} \partial \lambda_{u, v}} \approx \frac{Z \cdot \widetilde{\mu}_{r+u, l+v}-\widetilde{\mu}_{r, l} \widetilde{\mu}_{u, v}}{Z^{2}},
$$

where $0 \leq r+l \leq M, 0 \leq u+v \leq M$. Following the same procedure as in Section 4.1, the vector $\lambda^{*}=$ $\left(\lambda_{0,1}^{*}, \lambda_{1,0}^{*}, \ldots, \lambda_{0, M}^{*}, \lambda_{M, 0}^{*}\right)$ is found. The dimensionality of the optimization problem is $0.5\left(M^{2}+3 M\right)$, and $\lambda_{0,0}^{*}$ can be calculated from $\mathrm{A16}$ as $\lambda_{0,0}^{*}=\ln Z-1$. In comparison to the one-dimensional case, the range of the values of $\widetilde{\mu}_{r, l}$ becomes wider due to the larger dimensionality, so that the conditional number of the matrix $\left(H+\gamma^{(\ell)} \cdot \operatorname{diag}(H)\right)$ is even higher and the iteration might fail.

To approximate the moment values in A17) we truncate the infinite support and consider the subset $D_{x y}^{*}=D_{x}^{*} \times D_{y}^{*}$ instead. Again, we choose $D_{x y}^{*}$ such that the relative change of the dual function (A11) becomes smaller than the threshold $\delta_{\Psi}$. The approximation $\tilde{q}(x, y)$ of the marginal distribution $p_{., 0}(x, y, t)=P\left(X .(t)=x, X_{\circ}(t)=y\right)$ is then defined by the result $\lambda^{*}$ of the iteration procedure such that $p_{., \circ}(x, y, t) \approx \tilde{q}(x, y)$ if $(x, y) \in D_{x y}^{*}$ and $p_{., \circ}(x, y, t) \approx$ 0 if $(x, y) \notin D_{x y}^{*}$.

\section{Reconstruction of Distributions from Approximated Moments}

In the following we discuss the details of the reconstruction of marginal probability distributions based on solving the moment problem using the maximum entropy approach. We consider the three possibilities introduced in Section 5, weighted sum MCM, joint MCM and $M M$. We illustrate the details of all three approaches with examples.

Example 4. We consider the gene expression model (cf. Example 1) where we reconstruct the marginal distribution of protein molecules $P\left(X_{P}(t)=x\right)=p_{X_{P}}(x, t)$. The moments $\mu_{k}=E\left(X_{P}^{k}\right)$ and the corresponding conditional moments are obtained using the MCM and MM equations, for $k=0, \ldots, M+1$. In the case of joint $M C M$ and $M M$ we use the first $M$ moments' values as constraints in Eq. (2) and solve the maximum entropy optimization problem in Eq. (3). In both cases, the solution is given by a pair $\left(\lambda^{*}, D^{*}\right)$ of the parameter vector $\lambda^{*}$ and the truncated support $D^{*}$. The corresponding reconstructed distribution is defined as

$$
\tilde{q}(x)=\exp \left(-1-\sum_{k=0}^{M} \lambda_{k}^{*} x^{k}\right), x \in D^{*} .
$$


In order to apply the weighted sum MCM, we reconstruct the conditional distribution from the sequences $\mu_{P_{\text {off }}, k}$ and $\mu_{P_{\mathrm{on}}, k}$ that approximate the conditional moments $E\left(X_{P}^{k} \mid D_{\text {off }}=1\right)$ and $E\left(X_{P}^{k} \mid D_{\text {on }}=1\right)$. Here, $X_{P}$ corresponds to the number of proteins and the condition $D_{\text {off }}=1$ $\left(D_{\text {on }}=1\right)$ refers to the state of the gene. These sequences of moments are obtained using the MCM approach together with the approximation of the mode probabilities $p_{\text {off }}$ and $p_{\text {on }}$ (cf. Example 3). We solve the maximum entropy problem for each moment sequence and the reconstruction of marginal unconditional distribution is given by

$$
\tilde{q}_{w s M C M}(x)= \begin{cases}p_{\text {off }} \tilde{q}_{\text {off }}(x), & x \in D_{P_{\text {off }}}^{*} \backslash D_{P_{\text {on }}}^{*} \\ p_{\text {on }} \tilde{q}_{\text {on }}(x), & x \in D_{P_{\text {on }}}^{*} \backslash D_{P_{\text {off }}}^{*} \\ p_{\text {off }} \tilde{q}_{\text {off }}(x) & x \in D_{P_{\text {off }}}^{*} \cap D_{P_{\text {on }}}^{*}, \\ +p_{\text {on }} \tilde{q}_{\text {on }}(x) & \end{cases}
$$

where $\tilde{q}_{\text {off }}(x)$ and $\tilde{q}_{\text {on }}(x)$ are the reconstructions of the conditional distributions.

To reconstruct two-dimensional marginal distributions we numerically solve the two-dimensional maximum entropy problem as described in Section 4 . We illustrate how two-dimensional distributions are reconstructed through the following example where we apply the weighted sum MCM approach.

Example 5. We consider the exclusive switch system described in Section 5. The goal here is to reconstruct the two-dimensional marginal distribution

$P\left(X_{P_{1}}=x, X_{P_{2}}(t)=y\right)$ of proteins $P_{1}$ and $P_{2}$. We first approximate the mode probabilities $p_{1}=P(D N A=1)$, $p_{2}=P(D N A . P 1=1)$ and $p_{3}=P(D N A . P 2=1)$ (cf. Eq. A9P. In addition, the conditional moments

$$
\begin{aligned}
& \mu_{1 ; r, l}=E\left(X_{P_{1}}^{r} X_{P_{2}}^{l} \mid D N A=1\right) \\
& \mu_{2 ; r, l}=E\left(X_{P_{1}}^{r} X_{P_{2}}^{l} \mid D N A . P 1=1\right) \\
& \mu_{3 ; r, l}=E\left(X_{P_{1}}^{r} X_{P_{2}}^{l} \mid D N A . P 2=1\right)
\end{aligned}
$$

are approximated for $0 \leq r+l \leq M+1$, where $D N A=1$ refers to the case where the promoter is free and $D N A . P 1=1(D N A . P 2=1)$ to the case where a molecule of type $P_{1}$ (type $P_{2}$ ) is bound to the promoter. The constraints A13 for the maximum entropy problem are given by the elements of these three sequences for $0 \leq r+l \leq M$ and the corresponding solutions of the optimization problem are given by the pairs $\left(\lambda_{i}^{*}, D_{i}^{*}\right), i=\{1,2,3\}$. Then the reconstructed distribution is given by

$$
\begin{aligned}
& \tilde{q}_{w s M C M}(x, y)= \\
& \begin{cases}p_{1} \tilde{q}_{1}(x, y), & (x, y) \in D_{1}^{*} \backslash\left(D_{2}^{*} \cup D_{3}^{*}\right) \\
p_{2} \tilde{q}_{2}(x, y), & (x, y) \in D_{2}^{*} \backslash\left(D_{1}^{*} \cup D_{2}^{*}\right) \\
p_{3} \tilde{q}_{3}(x, y), & (x, y) \in D_{3}^{*} \backslash\left(D_{1}^{*} \cup D_{2}^{*}\right) \\
\sum_{i=1}^{2} p_{i} \tilde{q}_{i}(x, y), & (x, y) \in\left(D_{1}^{*} \cap D_{2}^{*}\right) \backslash D_{3}^{*}, \\
\sum_{i=\{1,3\}} p_{i} \tilde{q}_{i}(x, y), & (x, y) \in\left(D_{1}^{*} \cap D_{3}^{*}\right) \backslash D_{2}^{*}, \\
\sum_{i=2}^{3} p_{i} \tilde{q}_{i}(x, y), & (x, y) \in\left(D_{2}^{*} \cap D_{3}^{*}\right) \backslash D_{1}^{*}, \\
\sum_{i=1}^{3} p_{i} \tilde{q}_{i}(x, y), & (x, y) \in D_{1}^{*} \cap D_{2}^{*} \cap D_{3}^{*},\end{cases}
\end{aligned}
$$

where $\tilde{q}_{i}(x, y)=\exp \left(-1-\sum_{1 \leq r+l \leq M} \lambda_{r, l}^{*} x^{r} y^{l}\right)$.

\section{Case Studies}

Here we present detailed results of the reconstruction of the marginal distributions that were discussed in Sect. 5.

Gene Expression Model. We show the approximation error $\|\epsilon\|_{\infty}^{\%}$ for the reconstruction of both conditional and unconditional distributions for mRNA and protein in Table III where we use the first parameter set. Here, the first two columns refer to the approximation error of the conditional distributions for protein (mRNA) denoted by $P \mid D_{\text {off }}$ $\left(R \mid D_{\text {off }}\right)$ and $P \mid D_{\text {on }}\left(R \mid D_{\text {on }}\right)$. The last three columns refer to the reconstructions of the marginal distribution obtained using weighted sum MCM, joint MCM and $M M$, respectively.

We observe that the reconstruction is most accurate for the distribution of mRNA when the joint MCM method is applied with $M=7$. The distribution of protein molecules is reconstructed most accurately when $M M$ is applied with $M=7$. Please note that the large approximation errors of conditional distribution reconstructions may still provide an accurate reconstruction for the unconditional distribution because of the computation of a weighted sum that can average out individual deviations from the true probability value. For example, the reconstruction of the marginal distribution of proteins and mRNA with weighted sum MCM gives the smallest error when we use $M=3$ moments (6.5\% for proteins, $1.3 \%$ for mRNA), but the approximation errors of the corresponding conditional distributions are much larger.

The sensitivity of the optimization procedure can also influence the final result. The reconstruction that uses fewer degrees of freedom can provide an accurate solution since the distribution of the simple shape is able to explain the main behavior. At the same time, adding more moments into the consideration allows one to capture more details, but it may change the reconstruction drastically due to the sensitivity, and the corresponding approximation error can become larger. To the best of our knowledge, there exist no criteria that provide the number of moments that have to be considered such that adding more information does not

TABLE III: Approximation errors of mRNA and protein distributions reconstruction for gene expression system (first parameter set).

\begin{tabular}{cccccc}
\hline $\mathrm{M}$ & $P \mid D_{\text {off }}$ & $P \mid D_{\text {on }}$ & $P_{w s M C M}$ & $P_{j M C M}$ & $P_{M M}$ \\
\hline 3 & 15.1 & 26.7 & 6.5 & 10.5 & 10.2 \\
5 & 7.9 & 1.7 & 4.3 & 10.0 & 8.1 \\
7 & 18.4 & 2.8 & 12.5 & 7.9 & 3.3 \\
\hline $\mathrm{M}$ & $R \mid D_{\text {off }}$ & $R \mid D_{\text {on }}$ & $R_{w s M C M}$ & $R_{j M C M}$ & $R_{M M}$ \\
\hline 3 & 1.0 & 19.9 & 1.2 & 3.9 & 3.8 \\
5 & 0.5 & 4.6 & 1.1 & 0.2 & 0.4 \\
7 & 1.3 & 0.6 & 1.3 & 0.3 & 0.6 \\
\hline
\end{tabular}



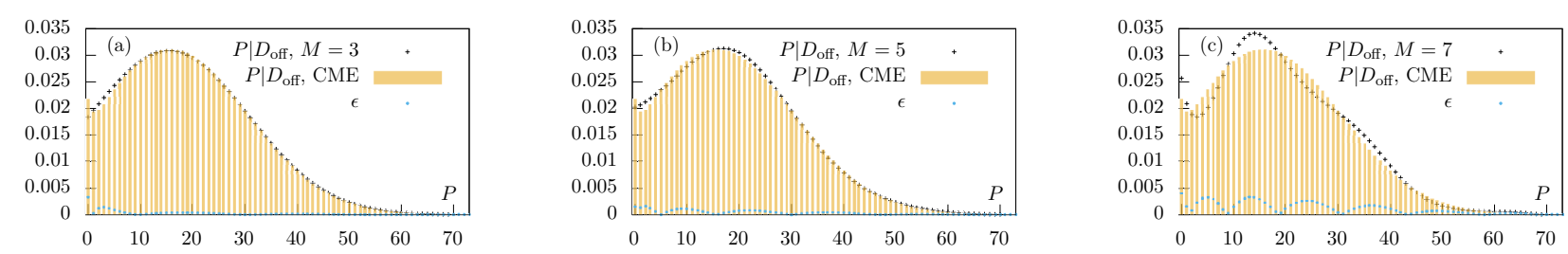

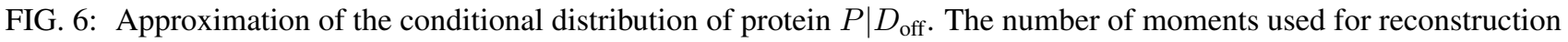
is $M=3(\mathrm{a}), M=5(\mathrm{~b})$ and $M=7$ (c).

greatly change the maximum entropy reconstruction. We show that in Fig. 6, where we plot the reconstructions of the conditional distribution $P \mid D_{\text {off }}$ and use $M \in\{3,5,7\}$. The reconstruction using $M=7$ moments has the largest approximation error, but it is able to capture the complex nature of the distribution by treating the point $P=0$ differently.

We note that the reconstruction results are generally quite similar for the approaches that are based on an approximation of the conditional and unconditional moments. However, the MCM approach has the advantage that the distribution of species such as DNA is very accurate, since they are directly available and are not reconstructed from the moments. A moment-based approach such as MM needs a large number of moments for an accurate reconstruction [2]. We also notice that the approximation of the conditional moments in the MCM method is less accurate than the approximation of the unconditional moments in the MM method (cf. Tables II and II) for this parameter set. Nevertheless, the reconstruction based on the conditional moments is in some cases more accurate, which means that the error is mostly due to the maximum entropy procedure.

An example of a two-dimensional distribution reconstruction is shown in Fig. 4. Here we present in addition the approximation errors for all three reconstruction methods in Tab. IV] both for conditional and marginal two-dimensional distributions of mRNA and protein. For the sake of readability we denote the reconstructed distribution by $\tilde{q}$ in the following tables. For instance, the approximation of the joint marginal distribution of $R$ and $P$ under the condition $D_{\text {off }}=1$ is denoted by $\tilde{q}_{D_{\text {off }}}$. We observe that the approximation error decreases when we make use of more moments.

The computation time for the reconstruction of the one-

TABLE IV: Approximation errors of two-dimensional distribution reconstruction for gene expression example (first parameter set).

\begin{tabular}{cccccc}
\hline $\mathrm{M}$ & $\tilde{q}_{D_{\text {off }}}$ & $\tilde{q}_{D_{\mathrm{on}}}$ & $\tilde{q}_{w s M C M}$ & $\tilde{q}_{j M C M}$ & $\tilde{q}_{M M}$ \\
\hline 3 & 59.1 & 48.9 & 58.1 & 62.1 & 62.0 \\
5 & 53.5 & 36.2 & 51.8 & 58.2 & 58.1 \\
7 & 28.0 & 29.1 & 28.1 & 24.7 & 29.1 \\
\hline
\end{tabular}

dimensional distribution for our MATLAB implementation (on a machine with the quad-core processor, $1.60 \mathrm{GHz}$ and $12 \mathrm{~GB}$ of RAM memory) is up to 0.3 seconds whereas the approximation of the two-dimensional distribution takes up to 15 seconds. The running time mainly depends on the support approximation procedure.

Next we consider the second parameter set. The approximation errors of the one-dimensional distributions are given in Table $\mathrm{V}$. The results of the reconstruction for this parameter set are worse than for the first one due to the more complex shape of the distribution. The weighted sum MCM provides the best result in all the cases (except for the reconstruction of the mRNA distribution when $M=3$ moments are used). In Fig. 7 we show the reconstructions both for protein and mRNA distribution obtained using $M=5$ moments. It can be seen that the maximum relative error $\|\epsilon\|_{\infty}^{\%}$ does not optimally describe the difference between the distribution shapes. For instance, a visual comparison of the reconstructed mRNA distributions reveals that the results obtained with joint MCM describes the shape better than the $M M$ based reconstruction though the relative error of joint MCM (> 100\%) is larger than that of $M M(45.6 \%)$.

The approximation errors of the two-dimensional distributions are given in Table VI It can be seen that the results are worse than those of the first parameter set and taking more moments into consideration does not give better results. Thus, the entropy maximization may not the best choice for the reconstruction of bi-modal distributions where the values of the peaks are of different orders of magnitude.

TABLE V: Approximation errors of reconstructed mRNA and protein distributions for the gene expression example (second parameter set).

\begin{tabular}{cccccc}
\hline $\mathrm{M}$ & $P \mid D_{\text {off }}$ & $P \mid D_{\text {on }}$ & $P_{w s M C M}$ & $P_{j M C M}$ & $P_{M M}$ \\
\hline 3 & 9.5 & 93.0 & 8.5 & 59.8 & 88.9 \\
5 & 21.3 & 70.3 & 20.1 & 23.1 & 71.6 \\
7 & 21.3 & 78.4 & 20.0 & $>100$ & 60.7 \\
\hline $\mathrm{M}$ & $R \mid D_{\text {off }}$ & $R \mid D_{\text {on }}$ & $R_{w s M C M}$ & $R_{j M C M}$ & $R_{M M}$ \\
\hline 3 & $>100$ & 10.7 & 85.9 & 25.1 & 71.5 \\
5 & 12.4 & 2.5 & 12.1 & $>100$ & 45.6 \\
7 & 12.4 & 1.3 & 12.2 & 46.1 & 33.7 \\
\hline
\end{tabular}




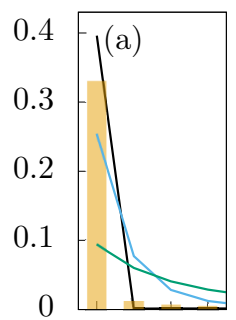

$\begin{array}{llll}0 & 1 & 2 & 3\end{array}$

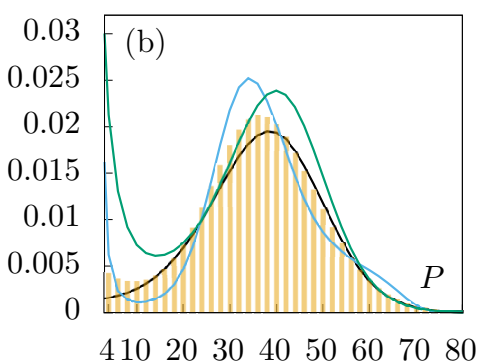

41020304050607080

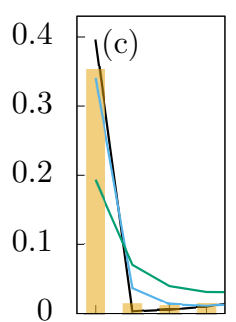

$\begin{array}{llll}0 & 1 & 2 & 3\end{array}$

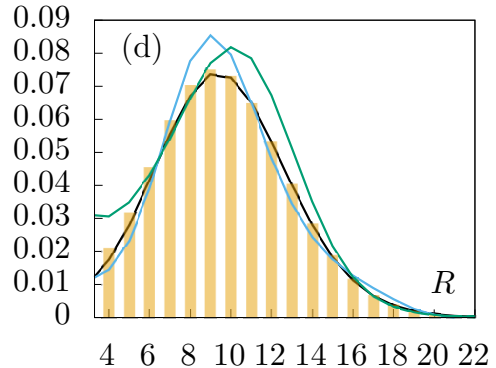

$4 \quad 6 \quad 8 \quad 10121416182022$

FIG. 7: Gene expression (second parameter set): approximations of the marginal distribution of protein (a), (b) and mRNA (c), (d) obtained using $M=5$ moments. The reconstructions are plotted with lines (black for weighted sum MCM, blue for joint MCM and green for $M M$ ) and the CME solution is plotted with bars. The plots (a) and (c) show in detail the region with molecular counts $\{0,1,2,3\}$.
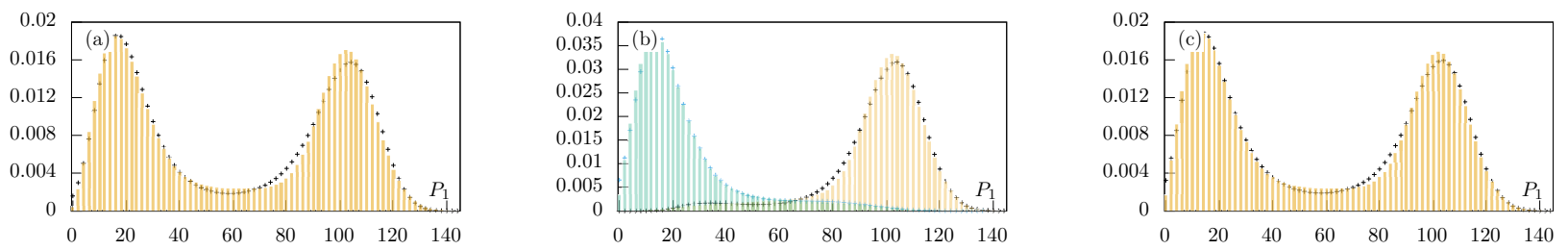

FIG. 8: Exclusive switch: approximations of the conditional distributions of protein $P_{1}$ where $D N A=1$ (a), $D N A . P_{1}=1, D N A . P_{2}=1$ (b) and the reconstruction of the marginal distribution (c). The solution of the CME is plotted with yellow bars and the reconstructions are plotted with black crosses (green bars and blue crosses are used for the conditional distribution (b) where $D N A \cdot P_{2}=1$ ). The reconstruction of the marginal distribution (c) is obtained using joint $M C M$ with $M=5$.

Exclusive Switch Model. Next we address the accuracy of the reconstruction of conditional and marginal distributions of the exclusive switch model introduced in Sect. 5. In Table VII the approximation errors are listed for the conditional distributions of the proteins where we condition on the three possible states of the promoter, i.e., $D N A=1$, $D N A . P_{1}=1$ or DNA.P $P_{2}=1$.

We observe that the approximation error $\|\epsilon\|_{\infty}^{\%}$ is minimal for both proteins $P_{1}$ and $P_{2}$ when the weighted sum $M C M$ approach is applied for all $M \in\{3,5,7\}$. Thus, for the exclusive switch system it is advantageous to approximate the marginal distributions by first reconstructing the conditional distributions and computing the weighted sum.

TABLE VI: Approximation errors of two-dimensional reconstruction for gene expression example (second parameter set).

\begin{tabular}{cccccc}
\hline $\mathrm{M}$ & $\tilde{q}_{D_{\mathrm{off}}}$ & $\tilde{q}_{D_{\mathrm{on}}}$ & $\tilde{q}_{w s M C M}$ & $\tilde{q}_{j M C M}$ & $\tilde{q}_{M M}$ \\
\hline 3 & 82.6 & 98.1 & 82.6 & 74.9 & 95.2 \\
5 & 83.6 & 83.4 & 83.6 & 76.0 & 88.1 \\
7 & 92.5 & 91.9 & 92.5 & 86.8 & 90.0 \\
\hline
\end{tabular}

In almost all cases the error decreases when more information about the moments is used. Because of the complex bimodal shape of the distributions, it is beneficial to consider higher-order moments. It is important to note also that the large value of the error $\left(\|\epsilon\|_{\infty}^{\%}>100\right)$ usually comes from the probabilities around the boundary points of the support $\left(x_{L}\right.$ or $\left.x_{R}\right)$. In the remaining parts of the support $D^{*}$ the reconstruction is accurate. For example, in Fig. 8 we show the reconstructions of both conditional (left and middle plots) and marginal (right plot) distributions of $P_{1}$. Here, the joint $M C M$ was used with $M=5$ to reconstruct the marginal

TABLE VII: Approximation errors for the distribution of proteins $P_{1}$ and $P_{2}$.

\begin{tabular}{|c|c|c|c|c|c|c|}
\hline$\Lambda$ & $1 \tilde{q}_{D N A}$ & $\tilde{q}_{D N A . P_{1}}$ & $\tilde{q}_{D N A . P_{2}}$ & $\tilde{q}_{w s M C M}$ & $\tilde{q}_{j M C M}$ & $\tilde{q}_{M M}$ \\
\hline 3 & $>100$ & 20.7 & $>100$ & $>100$ & $>100$ & $>100$ \\
\hline P. & 10.7 & 7.1 & 82.7 & 84.3 & $>100$ & $>100$ \\
\hline . & 5.7 & 7.8 & 79.5 & 80.5 & 6.6 & $>100$ \\
\hline & $>100$ & $>100$ & 40.8 & 41.5 & $>100$ & $>100$ \\
\hline 12 & 17.7 & $>100$ & 14.9 & 14.8 & 17.8 & $>100$ \\
\hline & 15.3 & 7.4 & 7.5 & 8.2 & 12.0 & 19.1 \\
\hline
\end{tabular}


distribution. The visual comparison reveals that the approximation nicely describes the bi-modal shape although the maximum relative error is large $\left(\|\epsilon\|_{\infty}^{\%}>100\right)$.

We also consider the conditional and marginal twodimensional distributions of proteins $P_{1}$ and $P_{2}$ in Table VIII. Again we condition on the state of the promoter region, e.g. $\tilde{q}_{D N A . P_{1}}$ corresponds to the joint distribution of proteins $P_{1}$ and $P_{2}$ when $D N A . P_{1}=1$.

The marginal distribution $P\left(X_{P_{1}}=x, X_{P_{2}}=y\right)$ is best approximated when the weighted sum MCM approach is applied and we see that better reconstructions are achieved with higher order moments. Generally, the MCM approach gives more accurate results, i.e., both weighted sum MCM and joint $M C M$ perform better than $M M$. We show the reconstructions of three conditional distributions in Fig. 9 for the case when $M=5$, where the plots refer to the conditions (from left to right) $D N A=1, D N A . P_{1}=1$ and $D N A . P_{2}=1$. The reconstruction of the marginal distribution obtained using weighted sum MCM is shown together with the approximation error in Fig. 9 (left and right plot). We observe that the approximation error is large in this case. In Fig. 9 we also plot the marginal distribution of $P_{2}$ where the mismatch for the first peak of the distribution can be explicitly seen. The reconstruction process for the exclusive switch model takes more time than for gene expression model because of a much larger support. The running time of the one-dimensional reconstruction is up to 3 seconds and in the two-dimensional case is up to 5 minutes. Again, here the bottleneck of the reconstruction procedure is the support approximation.

Thus, the idea of decomposing the Markov process into two parts, as done for the conditional moment equations, results in fewer equations and a more accurate description of the process. The weighted sum of mode probabilities and reconstructed conditional distributions seems to be particularly beneficial when systems exhibit complex behavior, such as in the exclusive switch model.

TABLE VIII: Two-dimensional conditional protein distributions (exclusive switch).

\begin{tabular}{ccccccc}
\hline $\mathrm{M}$ & $\tilde{q}_{D N A}$ & $\tilde{q}_{D N A . P_{1}}$ & $\tilde{q}_{D N A . P_{2}}$ & $\tilde{q}_{w s M C M}$ & $\tilde{q}_{j M C M}$ & $\tilde{q}_{M M}$ \\
\hline 3 & 69.089 & $>100$ & 49.3173 & 53.8828 & $>100$ & $>100$ \\
5 & 32.5258 & $>100$ & 47.1483 & 45.5285 & 24.4008 & $>100$ \\
7 & 19.7620 & $>100$ & 12.9727 & 14.2448 & 28.2168 & 26.2527 \\
\hline
\end{tabular}

[1] A. Ale, P. Kirk, and M. P. H. Stumpf. A general moment expansion method for stochastic kinetic models. The Journal of Chemical Physics, 138(17):174101, 2013.

[2] K. Bandyopadhyay, A. K. Bhattacharya, P. Biswas, and D. A. Drabold. Maximum entropy and the problem of moments: A stable algorithm. Physical Review E, 71(5):057701/1057701/4, 2005

[3] M. C. Guenther, Anton Stefanek, and J. T. Bradley. Moment closures for performance models with highly non-linear rates. In EPEW 2012, 9th European Performance Engineering Workshop, volume 7587 of Lecture Notes in Computer Science, pages 32-47, July 2012. ISBN 978-3-642-36780-9.

[4] C. A. Gómez-Uribe and G. C. Verghese. Mass fluctuation kinetics: Capturing stochastic effects in systems of chemical reactions through coupled mean-variance computations. The Journal of Chemical Physics, 126(2):024109, 2007. ISSN 0021-9606, 1089-7690. doi: 10.1063/1.2408422.
[5] J. Hasenauer, V. Wolf, A. Kazeroonian, and F.J. Theis. Method of conditional moments for the chemical master equation. Journal of Mathematical Biology, pages 1-49, 2013. ISSN 0303-6812.

[6] J. Hespanha. Moment closure for biochemical networks. In 3rd International Symposium on Communications, Control and Signal Processing, 2008. ISCCSP 2008, pages 142-147, 2008. doi: 10.1109/ISCCSP.2008.4537208.

[7] P. Milner, C. S. Gillespie, and D. J. Wilkinson. Moment closure approximations for stochastic kinetic models with rational rate laws. Mathematical Biosciences, 231(2):99-104, 2011. ISSN 0025-5564. doi: 10.1016/j.mbs.2011.02.006.

[8] A. Tari, M. Telek, and P. Buchholz. A simplified momentbased estimation method for extreme probabilities, infinite and positive cases, 2006. 

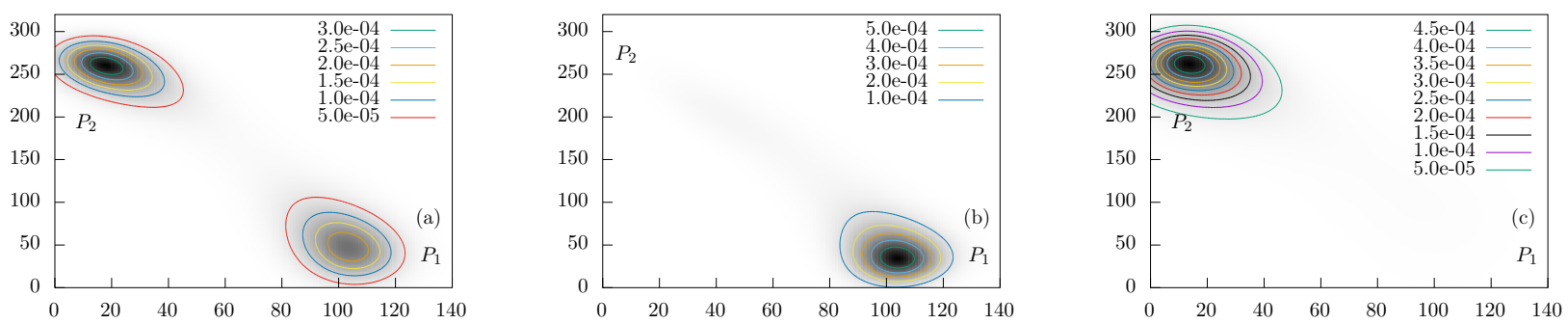

FIG. 9: Exclusive switch: the approximations of the conditional distributions of proteins $P_{1}$ and $P_{2}$ where $D N A=1$ (a), $D N A . P_{1}=1$ (b) and $D N A \cdot P_{2}=1$ (c). The reconstructions are obtained using $M=5$ moments.
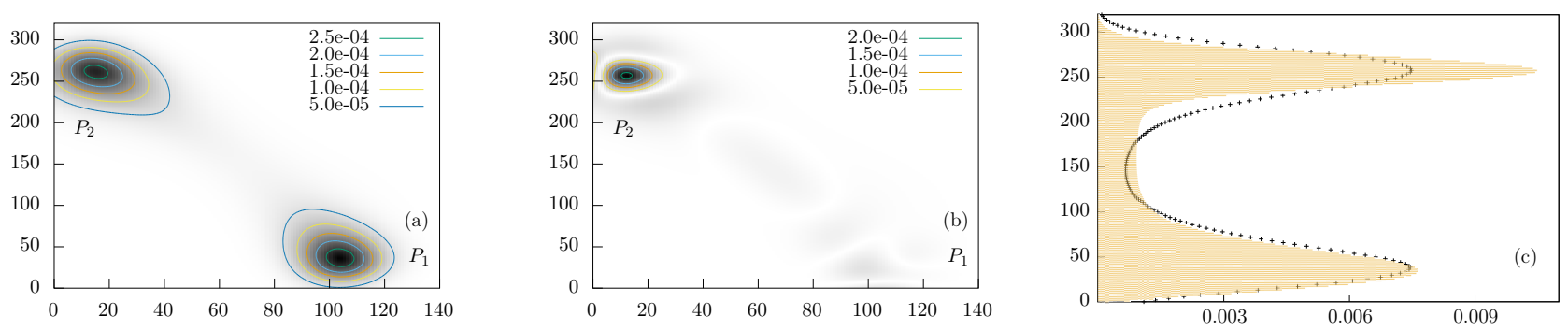

FIG. 10: Exclusive switch: the reconstruction of the marginal distribution of proteins $P_{1}$ and $P_{2}$ (a) obtained using weighted sum $M C M$ with $M=5$ moments and the corresponding approxmation error (b). The one-dimensional marginal distribution of $P_{2}$ (yellow bars) and the reconstruction (black dots) are shown in (c). 